\title{
A Case of Cruoricaptor ignavus Isolated From the Blood of a Patient With Ewing Sarcoma
}

Jongwon Oh, M.D. ${ }^{1}$, In Young Yoo, M.D. ${ }^{1}$, Dong Joon Song, M.T. ${ }^{1}$, Ji Won Lee, M.D. ${ }^{2}$, Yae-Jean Kim, M.D. ${ }^{2}$, Chang-Seok Ki, M.D. ${ }^{1}$, Nam Yong Lee, M.D. ${ }^{1}$, and Hee Jae Huh (D), M.D., Ph.D. ${ }^{1}$

Departments of ${ }^{1}$ Laboratory Medicine and Genetics and ${ }^{2}$ Pediatrics, Samsung Medical Center, Sungkyunkwan University School of Medicine, Seoul, Korea

\section{Dear Editor,}

Cruoricaptor ignavus, belonging to the family Flavobacteriaceae, is a gram-negative, non-motile, non-spore-forming coccoid- or coccobacilli-shaped bacterium first isolated from a human blood culture in 2012 and proposed as a novel genus and species [1]. With approval from Samsung Medical Center institutional review board (IRB; approval number: 2018-01-112), we report the second case worldwide of $C$. ignavus, isolated from the blood culture of a 16-year-old boy with Ewing sarcoma and identified by DNA target sequencing. The IRB waived the need for informed consent for this study.

The patient underwent wide excision of sarcoma and additional chemotherapy. On day 13 of chemotherapy, he developed fever with abdominal pain and visited the emergency room. His temperature was $38.3^{\circ} \mathrm{C}$, blood pressure was $116 / 59 \mathrm{mmHg}$, pulse rate was 67 beats per minute, and respiratory rate was 18 breaths per minute. The C-reactive protein concentration was $146.7 \mathrm{nmol} / \mathrm{L}$, and leukocyte count was $0.21 \times 10^{9} / \mathrm{L}$ with neutropenia $\left(0.07 \times 10^{9} / \mathrm{L}\right)$. Blood, urine, and stool cultures were performed, and cefepime was administered. Positive growth was observed in one of two sets of blood cultures after two days of incubation. Microscopic examination revealed gram-variable cocci or coccobacilli (Fig. 1), which grew as tiny, yellowish colonies on blood agar plates (Fig. 2).
The microorganism could not be identified using the GP, GN, and NH cards of the VITEK 2 system (bioMérieux, Marcy l'Etoile, France) or by matrix-assisted laser desorption/ionization time-offlight mass spectrometry (MALDI-TOF MS) with the Bruker MALDI Biotyper system (Bruker Daltonics GmbH, Leipzig, Germany). The VITEK MS system identified the organism as Alloiococcus otitis (99.9\% confidence), but Gram staining and colony morphology showed that this identification was not accurate.

To identify the strain, we performed $16 \mathrm{~S}$ ribosomal RNA (rRNA) target sequencing according to the CLSI guidelines [2]. Subregions of the 16S rRNA gene were amplified using the following primer pairs: forward, 4F: 5'-TTG GAG AGT TTG ATC CTG GCT C-3' and reverse, 534R: 5'-TAC CGC GGC TGC TGG CAC-3' and forward, 27F: 5'-AGA GTT TGA TCM TGG CTC AG3' and reverse, 801R: 5'-GGC GTG GAC TTC CAG GGT ATC T-3' [2]. The amplified sequence was compared with the GenBank (National Center for Biotechnology Information) database, using the basic local alignment search tool (BLAST) algorithm. The 16S rRNA sequence of the isolate exhibited 99.72\% (722/724 bp) similarity to $C$. ignavus (GenBank accession number NR_108875.1, Strain IMMIB L-12475 $)$. The second highest match was Epilithonimonas xixisoli with $87.07 \%$ (653/750 bp) similarity. When the sequence (724 bp) was submitted to the EzTaxon database v2.1 (http://www.ezbiocloud.net), the best-
Received: October 7, 2017

Revision received: April 2, 2018

Accepted: June 16, 2018

\section{Corresponding author: Hee Jae Huh}

(DiD https://orcid.org/0000-0001-8999-7561

Department of Laboratory Medicine and Genetics, Samsung Medical Center, Sungkyunkwan University School of Medicine, 81 Irwon-ro, Gangnam-gu, Seoul 06351, Korea

Tel: +82-2-3410-1836, Fax: +82-2-3410-2719, E-mail: pmhhj77@gmail.com

\section{(c) Korean Society for Laboratory Medicine}

This is an Open Access article distributed under the terms of the Creative Commons Attribution Non-Commercial License (http://creativecommons.org/licenses/by-nc/4.0) which permits unrestricted non-commercial use, distribution, and reproduction in any medium, provided the original work is properly cited. 


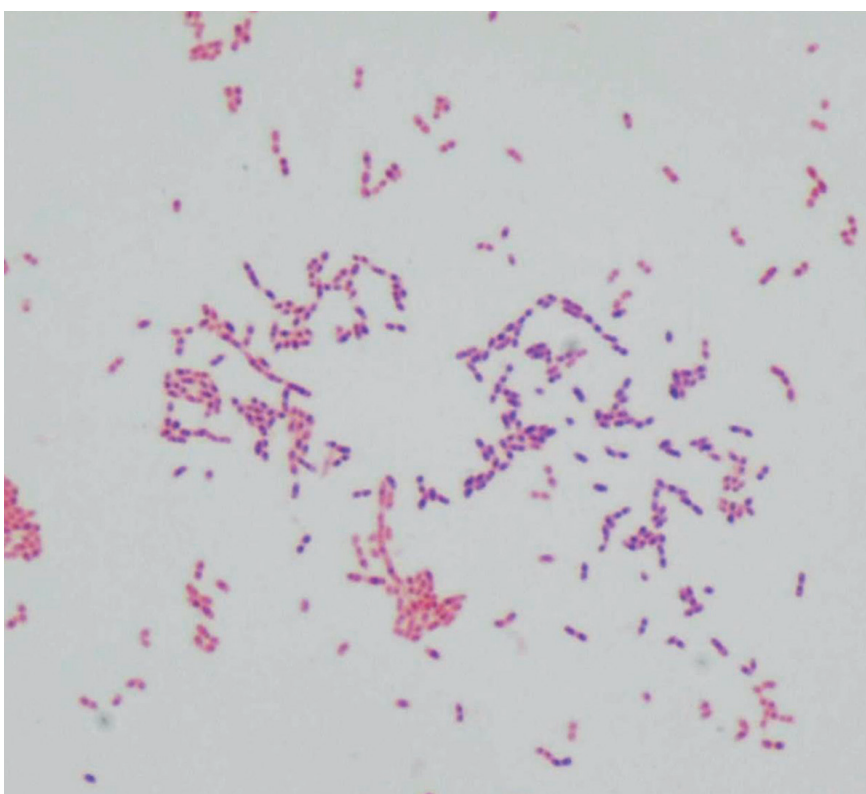

Fig. 1. Microscopic image of Cruoricaptor ignavus, showing gramvariable cocci or coccobacilli cells (Gram stain, $\times 1,000$ ).

matched strain was also C. ignavus (99.72\%), which was accepted as a final confirmation.

C. ignavus has rarely been isolated in clinical microbiology laboratories. Therefore, its pathogenic role remains unclear. In this case, the patient became afebrile after six days of antibiotic treatment, and follow-up culture showed no microorganism growth. The patient was discharged on the ninth day after admission. We cannot exclude the possibility that the isolate did not represent a true pathogen. However, considering that Flavobacteriaceae are a group of commensal bacteria and opportunistic pathogens, isolation of this bacterium in immunocompromised hosts may also represent a real infection scenario.

This case indicates that laboratory staff should be aware that C. ignavus can be misidentified or unidentified by commonly used identification systems. As Gram staining of $C$. ignavus is polymorphic and its morphology can appear both coccoid and coccobacilli-shaped, it may be challenging to distinguish $C$. ignavus based on colony and microscopic examination. Moreover, this pathogen could not be identified using the VITEK 2 system, and the VITEK MS system misidentified it as A. otitis, causing confusion. In a previous study, $A$. otitis was misidentified using Bruker Biotyper MALDI-TOF MS [3]. Although MALDI-TOF MS is commonly used in routine diagnostic laboratories, identification of new isolates is possible only if the spectral database contains peptide mass fingerprints of the type strains of specific genera/species/subspecies/strains $[4,5]$. C. ignavus was not in-

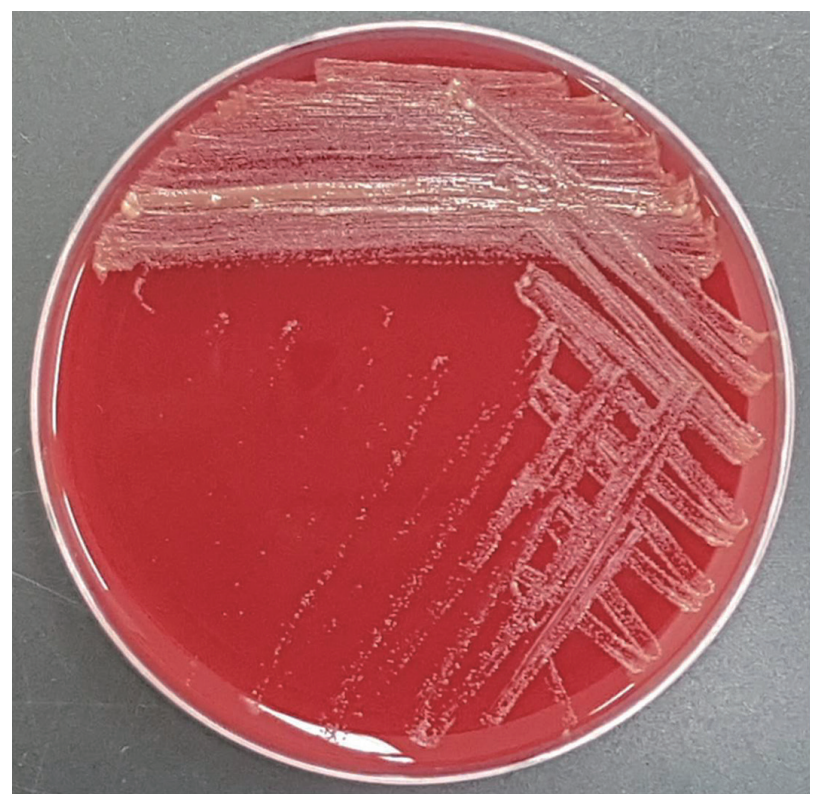

Fig. 2. Colony morphology of Cruoricaptor ignavus on blood agar plate.

cluded in the lists of identifiable species using the VITEK MS or Bruker Biotyper database.

To date, sequence-based typing remains the only available method for accurate identification of $C$. ignavus. Therefore, when an isolate is identified as A. otitis by MALDI-TOF MS and the microscopic morphology or biochemistry findings for that microorganism are inconsistent, confirmation via 16S rRNAbased gene sequencing is needed.

\section{Authors' Disclosures of Potential Conflicts of Interest}

No potential conflicts of interest relevant to this article were reported.

\section{REFERENCES}

1. Yassin AF, Inglis TJ, Hupfer H, Siering C, Schumann P, Busse HJ, et al. Cruoricaptor ignavus gen. nov., sp. nov., a novel bacterium of the family Flavobacteriaceae isolated from blood culture of a man with bacteraemia. Syst Appl Microbiol 2012;35:421-6.

2. CLSI. Interpretive criteria for identification of bacteria and fungi by DNA target sequencing; approved guideline. CLSI MM18-A. Wayne, PA: Clinical and Laboratory Standard Institute. 2008.

3. Jamal W, Albert MJ, Rotimi VO. Real-time comparative evaluation of bioMerieux VITEK MS versus Bruker Microflex MS, two matrix-assisted laser desorption-ionization time-of-flight mass spectrometry systems, for identification of clinically significant bacteria. BMC Microbiol 2014;14: 289. 
Oh J, et al.

Cruoricaptor ignavus from blood culture
ANNALS OF

LABORATORY MEDICINE
4. Biswas S and Rolain JM. Use of MALDI-TOF mass spectrometry for identification of bacteria that are difficult to culture. J Microbiol Methods 2013;92:14-24.
5. Singhal N, Kumar M, Kanaujia PK, Virdi JS. MALDI-TOF mass spectrometry: an emerging technology for microbial identification and diagnosis. Front Microbiol 2015;6:791. 